\title{
The Physiologic Response to Rescue Therapy with Vasopressin versus Epinephrine during Experimental Pediatric Cardiac Arrest
}

Julia Slovis ( $\square$ julia.slovis@gmail.com )

Children's Hospital of Philadelphia https://orcid.org/0000-0001-7773-1459

Ryan Morgan

Children's Hospital of Philadelphia

William Landis

Children's Hospital of Philadelphia

Anna L Roberts

Children's Hospital of Philadelphia

\section{Constantine Mavroudis}

Children's Hospital of Philadelphia

Yuxi Lin

Children's Hospital of Philadelphia

Tiffany Ko

Children's Hospital of Philadelphia

Vinay Nadkarni

Children's Hospital of Philadelphia

Robert A Berg

Children's Hospital of Philadelphia

Robert Sutton

Children's Hospital of Philadelphia

Todd J Kilbaugh

Children's Hospital of Philadelphia

\section{Research}

Keywords: Cardiac arrest, cardiopulmonary resuscitation, pediatrics, coronary perfusion pressure, cerebral blood flow, vasopressin, epinephrine

Posted Date: August 2nd, 2020

DOI: https://doi.org/10.21203/rs.3.rs-50678/v1 
License: (c) (i) This work is licensed under a Creative Commons Attribution 4.0 International License. Read Full License

Version of Record: A version of this preprint was published at Resuscitation Plus on December 1st, 2020. See the published version at https://doi.org/10.1016/j.resplu.2020.100050. 


\section{Abstract}

Background: While epinephrine is the mainstay of therapy during cardiopulmonary resuscitation, it is potentially detrimental to the cerebral vasculature and ineffective in certain populations. This study compares a rescue dose of vasopressin to a rescue dose of epinephrine after ineffective initial doses of epinephrine in diverse models of pediatric in-hospital cardiac arrest. 67 one- to three-month old female swine (10-30kg) in six experimental cohorts from one laboratory received hemodynamic-directed CPR, a resuscitation method where high quality chest compressions are provided and vasopressor administration is titrated to coronary perfusion pressure (CoPP) ${ }^{3} 20 \mathrm{mmHg}$. Vasopressors are given when CoPP is $<20 \mathrm{mmHg}$, in sequences of two doses of $0.02 \mathrm{mg} / \mathrm{kg}$ epinephrine separated by minimum oneminute, then a rescue dose of $0.4 \mathrm{U} / \mathrm{kg}$ vasopressin followed by minimum two-minutes. Invasive measurements were used to evaluate and compare the hemodynamic and neurologic effects of each vasopressor dose.

Results: Increases in CoPP and cerebral blood flow (CBF) were greater with vasopressin rescue than epinephrine rescue (CoPP: $+8.16[4.35,12.06] \mathrm{mmHg}$ vs. $+5.43[1.56,9.82] \mathrm{mmHg}, \mathrm{p}=0.022 ; \mathrm{CBF}:+14.58$ $[-0.05,38.12]$ vs. $+0.00[-0.77,18.24]$ perfusion units (PFU), $p=0.005)$. Twenty animals $(30 \%)$ failed to achieve CoPP ${ }^{3} 20 \mathrm{mmHg}$ after two doses of epinephrine; 9/20 (45\%) non-responders achieved CoPP ${ }^{3} 20$ $\mathrm{mmHg}$ after vasopressin. Among all animals, the increase in CBF was greater with vasopressin $(+14.58$ $[-0.58,38.12]$ vs. $0.00[-0.77,18.24]$ PFU, $p=0.005)$.

Conclusions: CoPP and CBF rose significantly more after rescue vasopressin than after rescue epinephrine. Importantly, CBF increased after vasopressin rescue, but not after epinephrine rescue. In the $30 \%$ that failed to meet CoPP of $20 \mathrm{mmHg}$ after two doses of epinephrine, $45 \%$ achieved target CoPP with a single rescue vasopressin dose.

\section{Background}

Pediatric in-hospital cardiac arrest (IHCA) occurs in 1.4-6\% of children admitted to pediatric intensive care units (Berg et al. 2016). Less than half of these children survive to hospital discharge and many have new functional morbidities post-arrest(Wolfe et al. 2019). During cardiopulmonary resuscitation (CPR), coronary perfusion pressure (CoPP), the difference between the aortic pressure and the right atrial (RA) pressure during the relaxation phase of chest compressions ("diastole"), is a major determinant of achieving return of spontaneous circulation (ROSC)(Paradis et al. 1990) and surviving to hospital discharge (Berg et al. 2018). Vasopressors are therefore given during CPR to increase systemic vascular resistance and thereby increase diastolic blood pressure (DBP) and CoPP(Berg et al. 2001). However, vasopressors may have adverse neurologic effects during CPR, with particular concern that epinephrine decreases cerebral blood flow(Gedeborg et al. 2000; Ristagno et al. 2007; Ristagno et al. 2008).

Our group developed and investigated the use of hemodynamic-directed CPR (HD-CPR), using systolic blood pressure-guided chest compression force and CoPP-guided vasopressor administration to improve 
outcomes (Chopra et al. 2016). In numerous pre-clinical studies, this HD-CPR strategy led to higher coronary and cerebral perfusion pressures, higher rates of survival, superior neurologic outcomes, and improved mitochondrial respiration in the heart and brain as compared to standard, guideline-based CPR (Friess et al. 2013; Lautz et al. 2019; Morgan et al. 2017; Naim et al. 2016; Sutton et al. 2013; Sutton et al. 2014). The vasopressor strategy employed in HD-CPR requires vasopressors to be administered in a protocolized manner if the CoPP is $<20 \mathrm{mmHg}$. An initial dose of $0.02 \mathrm{mg} / \mathrm{kg}$ epinephrine is given as dictated by CoPP, followed by a minimum duration of one minute, and a second dose of $0.02 \mathrm{mg} / \mathrm{kg}$ epinephrine if CoPP is $<20 \mathrm{mmHg}$. After an additional one-minute minimum duration, $0.4 \mathrm{Units} / \mathrm{kg}$ of vasopressin is administered if CoPP remains $<20 \mathrm{mmHg}$. Despite the efficacy of HD-CPR, the specific physiologic effects of vasopressin rescue have not been explicitly studied.

Therefore, the primary objective of this study was to compare the physiologic responses to a rescue dose (second dose) of epinephrine with a rescue dose of vasopressin. We hypothesized that CoPP and CBF would increase more following vasopressin than epinephrine(Ristagno et al. 2007). Additionally, we sought to characterize the physiologic response to vasopressin in a group of animals that failed to achieve CoPP ${ }^{3} 20 \mathrm{mmHg}$ after either of two doses of epinephrine (i.e., epinephrine non-responders). We hypothesized that many of these epinephrine "non-responders" would have increases in CoPP and CBF following a single dose of vasopressin.

\section{Materials And Methods}

\section{Study Design and Data Sources:}

This was a retrospective analytic study of data from laboratory experiments utilizing HD-CPR in porcine models of pediatric IHCA. Inclusion criteria were having HD-CPR as the resuscitation method and ${ }^{3} 2$ doses of epinephrine and ${ }^{3} 1$ dose of vasopressin administered. Animals did not require CBF data for inclusion, but were excluded if hemodynamic data were non-evaluable.

\section{Data Collection:}

The Children's Hospital of Philadelphia Institutional Animal Care and Use Committee approved all experimental protocols, which were conducted in accordance with the National Institutes of Health Guide for the Care and Use of Laboratory Animals. Comprehensive descriptions of animal preparation, anesthetic, and surgical methods are available in previous publications(Friess et al. 2013; Lautz et al. 2019; Morgan et al. 2016; Morgan et al. 2017; Morgan et al. 2018; Naim et al. 2016; Sutton et al. 2013; Sutton et al. 2014). Briefly, female Yorkshire swine were anesthetized and mechanically ventilated. To broadly study the pediatric age range, 1-month-old $(\sim 10 \mathrm{~kg})$ and 3-month-old $(\sim 30 \mathrm{~kg})$ swine were utilized. Vascular catheters were placed and high-fidelity pressure transducers were advanced to the RA, pulmonary artery (PA), and aorta for continuous hemodynamic measurements. Before and during the experimental protocol, the electrocardiogram (ECG), aortic pressure, RA pressure, PA pressure, pulse 
oximetry, and end-tidal carbon dioxide (ETCO2) values and waveforms were displayed and recorded. Coronary perfusion pressure was automatically calculated and displayed in real time by subtracting the RA pressure from the aortic pressure(Morgan et al. 2017). A CPR quality-recording defibrillator (Zoll R Series Plus; Zoll Medical Corporation) was used during CPR and recorded chest compression rate (per minute) and depth (centimeters). A subset of animals underwent invasive neuromonitoring with a PeriFlux laser Doppler (Perimed, Inc.) monitor to measure CBF, located in the superficial cerebral cortex.

\section{Experimental Protocol:}

Injury Period: Animals underwent one of the following injuries:

1) Primary ventricular fibrillation (VF) cardiac arrest (3-month-old swine): VF was electrically induced and left untreated for seven minutes, followed by a minimum of 10 minutes of HD-CPR and defibrillation(Friess et al. 2013; Naim et al. 2016).

2) Asphyxia-associated cardiac arrest (1- and 3-month-old swine): Asphyxia was induced by clamping the endotracheal tube for 7 minutes, following which VF was induced and HD-CPR commenced(Lautz et al. 2019; Morgan et al. 2017; Sutton et al. 2013; Sutton et al. 2014). The induction of VF ensured a consistent 10-minute CPR period as effective CPR after asphyxia-induced cardiac arrest alone typically results in ROSC within 2-4 minutes(Berg et al. 1999; Berg et al. 2000).

3) Lipopolysaccharide (LPS)-induced shock-associated IHCA (3-month-old swine): Animals received 45 minutes of an intravenous LPS infusion to induce shock, followed by induction of VF to ensure an adequate cardiac arrest period in which to study HD-CPR(Morgan et al. 2018). A subset of these animals received nitric oxide (iNO) therapy.

Resuscitation Period: In all subjects, chest compressions were provided with a target rate of 100 per minute guided by metronome. Per our established HD-CPR algorithm (Figure 1), chest compression depth was titrated to maintain a systolic blood pressure (SBP) of $90 \mathrm{mmHg}$ for $10 \mathrm{~kg}$ swine and $100 \mathrm{mmHg}$ for $30 \mathrm{~kg}$ swine. Vasopressors were given by protocol, as needed, to maintain a goal CoPP of ${ }^{3} 20 \mathrm{mmHg}$. Beginning two minutes into CPR, epinephrine was administered $(0.02 \mathrm{mg} / \mathrm{kg}$ as recommended for swine models(Berg et al. 1994; Brown et al. 1987; Gonzalez et al. 2014)) if CoPP $<20$ mmHg during mid-diastole for at least three consecutive chest compressions. If CoPP was $<20 \mathrm{mmHg}$ one minute after first epinephrine dose or at any subsequent time, an additional dose of epinephrine was administered. If CoPP was $<20 \mathrm{mmHg}$ one minute after second epinephrine dose or any subsequent time, vasopressin $(0.4$ 
$\mathrm{U} / \mathrm{kg}$ ) was administered. The cycle restarted two minutes following vasopressin, with these minimum intervals based on the peak effect of each vasopressor.

\section{Data Collection and Processing:}

All physiologic measurements were recorded at $1000 \mathrm{~Hz}$ (PowerLab, ADInstruments, Inc.). Vasopressor administration was recorded in real-time. The one minute preceding each vasopressor dose, the one minute following each epinephrine dose, and the two minutes following each vasopressin dose were divided into 15-second data epochs. Mean values for each physiologic parameter were calculated for each epoch. Data were analyzed from only the first cycle of vasopressors (i.e., epinephrine, epinephrine, vasopressin) for each experiment to minimize confounding. For comparison of physiologic response between rescue vasopressors after initial epinephrine dose failed to sustain the target CoPP, the second dose of epinephrine was compared to the rescue vasopressin dose.

\section{Statistical Analyses:}

The primary analysis compared the physiologic change following vasopressin to that observed following the second dose of epinephrine. The primary outcomes were changes in CoPP and CBF. All measurements were treated as non-normally distributed after a Shapiro-Wilk analysis was performed and were reported as medians with interquartile ranges and compared using non-parametric analyses. The change in each physiologic variable from mean of the four pre-vasopressor epochs to highest postvasopressor epoch (four epochs following epinephrine and eight epochs following vasopressin) was calculated and these values were compared between vasopressin and epinephrine with Wilcoxon signedrank tests.

For the secondary analyses, an epinephrine CoPP threshold non-responder cohort was defined a priori as animals that failed to have any 15 -second mean CoPP ${ }^{3} 20 \mathrm{mmHg}$ after either dose of epinephrine. These subjects were characterized as vasopressin responders if their CoPP was ${ }^{3} 20 \mathrm{mmHg}$ in any 15 -second epoch during the two minutes after vasopressin administration. The above analyses were repeated in subgroups of epinephrine responders, epinephrine non-responders, and in epinephrine non-responders that responded to vasopressin.

In prospectively planned supplemental analyses, the change in CoPP following the second dose of epinephrine to a priori response targets $\left({ }^{3} 3 \mathrm{mmHg} ;{ }^{3} 5 \mathrm{mmHg} ;{ }^{3} \mathrm{mmHg} ;{ }^{3} 10 \mathrm{mmHg}\right.$ ) were utilized as alternative definitions of epinephrine responsiveness. The number of responders and non-responders in each of these categories was summarized, as were the number of vasopressin responders (according to the same definition) among each epinephrine non-responder population. 


\section{Results}

\section{Physiologic Responses in the Overall Population}

Data from 98 experiments were compiled and evaluated for study inclusion. After exclusion of 31 subjects that were epinephrine responders and did not require three vasopressor doses, 67 remained for analysis (Figure 2). In the overall cohort $(n=67)$, the mean baseline CBF prior to cardiac arrest was $348.16 \pm 38$ PFU (SEM), which dropped to 0 PFU in all animals at the start of CPR. There was a significant increase in CoPP and CBF after both doses of epinephrine and after vasopressin (Supplemental Table 1; Figures 3 and 4). Vasopressin generated a greater rise in CoPP $(+8.16[4.35$, $12.06] \mathrm{mmHg}$ vs. $+5.43[1.56,9.82] \mathrm{mmHg} ; \mathrm{p}=0.22)$, mean PA pressure $(+4.05[0.42,7.80] \mathrm{mmHg}$ vs. $+5.90[1.98,13.82] \mathrm{mmHg}, \mathrm{p}<0.001)$ and $\mathrm{CBF}(+14.58[-0.05,38.12]$ PFU vs. $+0.00[-0.77,18.24]$ PFU, $\mathrm{p}=0.005)$ relative to the second dose of epinephrine (Table 1$)$.

\section{Physiologic Responses among Epinephrine Non-Responders}

In 20/67 (29.9\%) experiments, neither dose of epinephrine resulted in any epoch with CoPP ${ }^{3} 20 \mathrm{mmHg}$. Nine of these $20(45 \%)$ achieved a CoPP of ${ }^{3} 20 \mathrm{mmHg}$ within 2 minutes after a single dose of rescue vasopressin. Among these 20 epinephrine non-responders, the change in CBF of $-0.28[-2.33,6.91]$ PFU after the epinephrine rescue was significantly less than after vasopressin rescue +14.58 [3.55 - 34.90] PFU ( $p=0.045)$ (Table 1; Figure 4). The change in CoPP with was $+2.76[1.28,6.58] \mathrm{mmHg}$ with epinephrine rescue and $+7.64[4.86,11.02] \mathrm{mmHg}$ with vasopressin $(p=0.22)$ (Table 1 ; Figure $3 c)$.

\section{Alternative characterization of epinephrine responsiveness}

Alternative definitions of vasopressin responsiveness according to change in CoPP attributable to the second dose of epinephrine are depicted in Supplemental Table 2. Thirty-one of 67 (46\%) animals showed a rise of ${ }^{3} 3 \mathrm{mmHg}$ after the second dose of epinephrine while $36 / 67$ (54\%) did not. Of the 36 who did not meet the goal rise of ${ }^{3} 3 \mathrm{mmHg}, 20(56 \%)$ rose ${ }^{3} 3 \mathrm{mmHg}$ after vasopressin. In this population of epinephrine non-responders $(n=36)$, CBF fell $-0.003[-0.9,14.60]$ PFU after the second dose of epinephrine compared to rising $+10.6[0.08,37.4]$ PFU after rescue vasopressin $(p=0.01) .21 / 67(31 \%)$ saw an increase in CoPP ${ }^{3} 5 \mathrm{mmHg}$ after the second dose of epinephrine, whereas 46/67 (69\%) did not meet this goal. In this population $(n=46), C B F$ rose $+0.07[-0.7,16.3]$ PFU after the second dose of epinephrine compared to $+21.2[0.04,39.20]$ PFU after vasopressin $(p=0.002)$.

\section{Discussion}

In this large analytic study of swine treated with HD-CPR across several diverse pathophysiologic models of IHCA (i.e., asphyxia, ventricular fibrillation, endotoxemia), the increases in coronary perfusion pressure 
and importantly, cerebral blood flow were consistently greater after vasopressin rescue than after rescue with a second dose of epinephrine. In the $30 \%$ of animals who failed to achieve the a priori hemodynamic goal CoPP of $20 \mathrm{mmHg}$ after two doses of epinephrine (i.e. epinephrine non-responders), CBF increased more and nearly half attained the CoPP goal of $20 \mathrm{mmHg}$ after a single "rescue" dose of vasopressin (Table 1). While prior published data do not support the routine use of vasopressin as a substitute for epinephrine or combined with epinephrine(Panchal et al. 2019) our data support the consideration of vasopressin as a potential rescue therapy when epinephrine does not achieve adequate CoPP response. The use of rescue vasopressin in this manner may raise CBF more than providing another dose of epinephrine.

In the overall cohort, vasopressin had significantly greater effect on CoPP, a value that directly correlates with ROSC(Paradis et al. 1990), than the preceding rescue dose of epinephrine. The magnitude of the difference in CoPP response between vasopressin and epinephrine was greatest in epinephrine nonresponders $(+7.64[4.86,11.02] \mathrm{mmHg}$ vs. $+2.76[1.28,6.58] \mathrm{mmHg})$, though this difference did not reach statistical significance. This improvement in CoPP is consistent with prior work showing significantly higher CoPP after a combination of vasopressin and epinephrine than after either medication alone(Mayr et al. 2001).

To ensure these findings were robust and potentially translatable to a practical clinical setting, we sought to further characterize the response to the second dose of epinephrine and offer alternative definitions of epinephrine responsiveness based upon thresholds of CoPP rising ${ }^{3} 3 \mathrm{mmHg},{ }^{3} 5 \mathrm{mmHg},{ }^{3} 7 \mathrm{mmHg}$, or ${ }^{3} 10$ $\mathrm{mmHg}$ after the second dose of epinephrine (Supplemental Table 2). Since less than half of animals $(31 / 67,46 \%)$ achieved an increase in CoPP of ${ }^{3} 3 \mathrm{mmHg}$, it is clear that a population of epinephrine nonresponders exists even with alternative definitions. Cumulatively, these data demonstrate heterogeneity in the hemodynamic response to epinephrine and that some animals with a poor response to epinephrine do, in fact, respond to vasopressin (Figure 3c).

Vasopressin also increased CBF more than the rescue dose of epinephrine in all responder groups (Table 1). The second "rescue" dose of epinephrine did not increase median CBF (Figure 4). Vasopressor effects on the cerebral vasculature are complex and highly variable, with high doses of vasopressors at risk to decrease rather than increase perfusion to critical areas of brain(Thorup et al. 2019). Epinephrine, a potent mixed alpha- and beta-adrenergic agonist and the mainstay of vasopressor therapy during CPR(de Caen et al. 2015), has potential detrimental cerebral effects, and higher rescue doses of epinephrine have been shown to induce cerebral vasoconstriction in animal studies(Gedeborg et al. 2000). These effects can include decreased cerebral oxygenation(Ristagno et al. 2007; Ristagno et al. 2009) and impaired cerebral microvascular blood flow(Ristagno et al. 2009). In addition, the large, randomized clinical trial PARAMEDIC II suggested that cardiac arrest survivors treated with epinephrine had more severe neurologic impairment than those without epinephrine (Perkins et al. 2018).

Vasopressin may have less adverse effects on the cerebral microvasculature than epinephrine. Previous porcine translational models of cardiac arrest have demonstrated greater $\mathrm{CBF}$, higher cerebral $\mathrm{pH}$, lower 
PCO2, and lower cerebral oxygen extraction after vasopressin vs. epinephrine(Prengel et al. 1996), and improved rates of survival with good neurologic outcomes(Wenzel et al. 2000b). Other porcine studies have shown vasopressin to be more effective in raising cerebral blood flow either alone(Wenzel et al. 2000a) or in combination with epinephrine(Voelckel et al. 2000; Voelckel et al. 2002). A large, randomized clinical trial showed that vasopressin combined with epinephrine and steroids, compared to epinephrine alone, may increase survival to hospital discharge with favorable neurological status(Mentzelopoulos et al. 2013). However, vasopressin remains a Class Ilb recommendation in adults and Class Indeterminate recommendation in children by current resuscitation guidelines(Panchal et al. 2019).

Through activation of V1a receptors on smooth muscle cells, vasopressin may facilitate a more pronounced blood flow shift from peripheral to vital organs. These mechanistic differences, coupled with superior systemic hemodynamics, may explain the greater CBF changes following vasopressin relative to epinephrine, and these may be more pronounced in combination with epinephrine, specifically as a rescue therapy. Cumulatively, these existing studies and the present data support the conduct of head-to-head prospective studies comparing CBF and neurologic outcome with rescue vasopressin compared to rescue standard dose epinephrine (e.g. usual care).

Most pediatric cardiac arrests occur in ICUs and many of these patients have arterial catheters in place at the time of arrest(Berg et al. 2013), thus one could determine which patients fail physiologic response to initial epinephrine boluses. Prior negative adult human randomized controlled trials have randomized initia/vasopressin therapy vs epinephrine therapy(Wenzel et al. 2004) or combined initial vasopressinepinephrine vs epinephrine therapy(Gueugniaud et al. 2008), but meta-analyses of combined routine vasopressin-epinephrine therapies have only shown promise for out of hospital adult asystolic arrest(Mentzelopoulos et al. 2012). Vasopressin in these trials was administered in a standardized manner without regard to hemodynamic responses, so it is possible that there would be increased efficacy when administered to achieve diastolic blood pressure goals that are associated with improved outcomes(Berg et al. 2018). Future work should compare rescue epinephrine and vasopressin head-tohead in a physiology-directed fashion, stratified by initial host response to epinephrine.

This study has limitations. First, its pre-clinical nature could limit translatability to the bedside. However, experiments were conducted in established translational porcine models of cardiac arrest, subjects underwent consistent and standardized injuries, received consistent and highly protocolized resuscitations to minimize variability, and had closed systems of invasive monitoring for hemodynamic and neurologic data collection, with paired analyses allowing effect comparisons within a single subject. In addition, these laboratory models are relevant to patients in the ICU setting with arterial catheters in place at the time of cardiac arrest. Second, as this was an observational analysis of animals receiving HD-CPR, we did not compare vasopressin "rescue" to "rescue" with a third dose of epinephrine. Because these animals had less response to the second dose of epinephrine than to the first dose (Figure 3a, Figure 4), and subsequent boluses of epinephrine have diminishing effects(Nosrati et al. 2019; Wenzel et al. 1999), it is likely that the differences would have been more marked if we compared a third dose of epinephrine to vasopressin as the third vasopressor dose. 


\section{Conclusions}

In translational models of CPR, rescue doses of vasopressin increased coronary perfusion pressure and cerebral blood flow greater than rescue doses of epinephrine. Among the $30 \%$ of animals who failed to meet a CoPP threshold of $20 \mathrm{mmHg}$ after two standard epinephrine doses (epinephrine non-responders), $\mathrm{CBF}$ increased more with vasopressin than epinephrine and $45 \%$ attained the CoPP goal of $20 \mathrm{mmHg}$ after a single "rescue" dose of vasopressin. These findings suggest that rescue doses of vasopressin may potentially be beneficial for selected patients who did not respond adequately to epinephrine during CPR.

\section{Declarations}

\section{Ethics approval and consent to participate:}

The Children's Hospital of Philadelphia Institutional Animal Care and Use Committee approved all experimental protocols, which were conducted in accordance with the National Institutes of Health Guide for the Care and Use of Laboratory Animals. Institutional Animal Care and Use Committee (IACUC) Protocol \#'s:

IAC 16-001023

IAC 14-00112

Consent for publication: Not applicable

\section{Availability of data and material}

The datasets used and/or analysed during the current study are available from the corresponding author on reasonable request

\section{Competing interests}

Morgan receives grant funding from the $\mathrm{NIH}$ and serves on the American Heart Association (AHA) Emergency Cardiovascular Care Committee. Dr. Berg receives grant funding from the NIH. Dr. Sutton receives grant funding from the $\mathrm{NIH}$, serves on the AHA Emergency Cardiovascular Care Committee, and is Vice Chair of the AHA Get with the Guidelines-Resuscitation Pediatric Task Force. Dr. Kilbaugh receives grant funding from the National Institutes of Health $(\mathrm{NIH})$ and the Department of Defense. Dr Nadkarni receives unrestricted grant funding from the NIH, AHRQ, Zoll Medical, American Heart Association, Laerdal Medical, Nihon Kohden, Inc and serves as a volunteer committee member for the American Heart Association, International Liaison Committee on Resuscitation, Citizen CPR Foundation, and Society for Critical Care Medicine.

\section{Funding}


Financial support was provided through Russell Raphaely Endowed Chair funds at the Children's Hospital of Philadelphia and grant funding through the NIH National Heart, Lung, and Blood Institute (R01 HL141386 \& K23HL148541) and Eunice Kennedy Shriver National Institute for Child Health and Human Development (R21HD089132).

\section{Authors' contributions. All authors read and approved the final manuscript.}

JS analyzed and interpreted the data, performed statistical analyses, and wrote the manuscript.

RWM analyzed and interpreted the data, performed statistical analyses, and contributed major changes and revisions to the manuscript.

WPL collected and analyzed all data, along with data interpretation and manuscript revision.

ALR collected and analyzed data and revised the manuscript.

AMM collected and analyzed data and revised the manuscript.

CDM collected and analyzed data and revised the manuscript.

YL collected and analyzed data.

TK collected and analyzed data and revised the manuscript.

VMN collected and analyzed data and provided multiple rounds of insights to edit the concept and content of the manuscript.

RAB analyzed data and provided multiple rounds of insights to edit the concept and content of the manuscript.

RMS analyzed data and provided multiple rounds of insights to edit the concept and content of the manuscript.

TJK analyzed and interpreted the data, performed statistical analyses, and contributed major changes and revisions to the manuscript.

Acknowledgements: Not applicable.

Authors' information (optional)

Robert A Berg is the co-founding director of the Center for Pediatric Resuscitation and a Russell Raphaley Endowed Chair as the division chief of Critical Care Medicine within the Department of Anesthesiology and Critical Care Medicine.

\section{References}


Berg RA, Hilwig RW, Kern KB, Babar I, Ewy GA (1999) Simulated mouth-to-mouth ventilation and chest compressions (bystander cardiopulmonary resuscitation) improves outcome in a swine model of prehospital pediatric asphyxial cardiac arrest. Crit Care Med 27(9):1893-9 doi:10.1097/00003246199909000-00030 Berg RA, Hilwig RW, Kern KB, Ewy GA (2000) "Bystander" chest compressions and assisted ventilation independently improve outcome from piglet asphyxial pulseless "cardiac arrest". Circulation 101(14):1743-8 doi:10.1161/01.cir.101.14.1743 Berg RA, Nadkarni VM, Clark AE, et al. (2016) Incidence and Outcomes of Cardiopulmonary Resuscitation in PICUs. Crit Care Med 44(4):798-808 doi:10.1097/CCM.0000000000001484 Berg RA, Otto CW, Kern KB, et al. (1994) High-dose epinephrine results in greater early mortality after resuscitation from prolonged cardiac arrest in pigs: a prospective, randomized study. Crit Care Med 22(2):282-90 doi:10.1097/00003246-199402000-00020 Berg RA, Sanders AB, Kern KB, et al. (2001) Adverse hemodynamic effects of interrupting chest compressions for rescue breathing during cardiopulmonary resuscitation for ventricular fibrillation cardiac arrest. Circulation 104(20):2465-70 doi:10.1161/hc4501.098926 Berg RA, Sutton RM, Holubkov R, et al. (2013) Ratio of PICU versus ward cardiopulmonary resuscitation events is increasing. Crit Care Med 41(10):2292-7 doi:10.1097/CCM.0b013e31828cf0c0 Berg RA, Sutton RM, Reeder RW, et al. (2018) Association Between Diastolic Blood Pressure During Pediatric In-Hospital Cardiopulmonary Resuscitation and Survival. Circulation 137(17):1784-1795 doi:10.1161/CIRCULATIONAHA.117.032270 Brown CG, Werman HA, Davis EA, Hobson J, Hamlin RL (1987) The effects of graded doses of epinephrine on regional myocardial blood flow during cardiopulmonary resuscitation in swine. Circulation 75(2):491-7 doi:10.1161/01.cir.75.2.491 Chopra AS, Wong N, Ziegler CP, Morrison LJ (2016) Systematic review and meta-analysis of hemodynamic-directed feedback during cardiopulmonary resuscitation in cardiac arrest. Resuscitation 101:102-7 doi:10.1016/j.resuscitation.2016.01.025 de Caen AR, Berg MD, Chameides L, et al. (2015) Part 12: Pediatric Advanced Life Support: 2015 American Heart Association Guidelines Update for Cardiopulmonary Resuscitation and Emergency Cardiovascular Care. Circulation 132(18 Suppl 2):S526-42 doi:10.1161/CIR.0000000000000266 Friess SH, Sutton RM, Bhalala U, et al. (2013) Hemodynamic directed cardiopulmonary resuscitation improves short-term survival from ventricular fibrillation cardiac arrest. Crit Care Med 41(12):2698-704 doi:10.1097/CCM.0b013e318298ad6b Gedeborg R, Silander HC, Ronne-Engstrom E, Rubertsson S, Wiklund L (2000) Adverse effects of high-dose epinephrine on cerebral blood flow during experimental cardiopulmonary resuscitation. Crit Care Med 28(5):1423-30 doi:10.1097/00003246-200005000-00028 Gonzalez R, Urbano J, Botran M, et al. (2014) Adrenaline, terlipressin, and corticoids versus adrenaline in the treatment of experimental pediatric asphyxial cardiac arrest. Pediatr Crit Care Med 15(6):e280-7 doi:10.1097/PCC.0000000000000127 Gueugniaud PY, David JS, Chanzy E, et al. (2008) Vasopressin and epinephrine vs. epinephrine alone in cardiopulmonary resuscitation. N Engl J Med 359(1):21-30 doi:10.1056/NEJMoa0706873 Lautz AJ, Morgan RW, Karlsson M, et al. (2019) Hemodynamic-Directed Cardiopulmonary Resuscitation Improves Neurologic Outcomes and Mitochondrial Function in the Heart and Brain. Crit Care Med 47(3):e241-e249 doi:10.1097/CCM.0000000000003620 Mayr VD, Wenzel V, Voelckel WG, et al. (2001) Developing a vasopressor combination in a pig model of adult asphyxial cardiac arrest. Circulation 104(14):1651-6 doi:10.1161/hc3901.095896 Mentzelopoulos SD, Malachias S, Chamos C, et al. (2013) Vasopressin, steroids, and epinephrine and neurologically favorable survival after 
in-hospital cardiac arrest: a randomized clinical trial. JAMA 310(3):270-9 doi:10.1001/jama.2013.7832 Mentzelopoulos SD, Zakynthinos SG, Siempos I, Malachias S, Ulmer H, Wenzel V (2012) Vasopressin for cardiac arrest: meta-analysis of randomized controlled trials. Resuscitation 83(1):32-9 doi:10.1016/j.resuscitation.2011.07.015 Morgan RW, French B, Kilbaugh TJ, et al. (2016) A quantitative comparison of physiologic indicators of cardiopulmonary resuscitation quality: Diastolic blood pressure versus end-tidal carbon dioxide. Resuscitation 104:6-11 doi:10.1016/j.resuscitation.2016.04.004 Morgan RW, Kilbaugh TJ, Shoap W, et al. (2017) A hemodynamic-directed approach to pediatric cardiopulmonary resuscitation (HD-CPR) improves survival. Resuscitation 111:41-47

doi:10.1016/j.resuscitation.2016.11.018 Morgan RW, Sutton RM, Karlsson M, et al. (2018) Pulmonary Vasodilator Therapy in Shock-associated Cardiac Arrest. Am J Respir Crit Care Med 197(7):905-912 doi:10.1164/rccm.201709-18180C Naim MY, Sutton RM, Friess SH, et al. (2016) Blood Pressure- and Coronary Perfusion Pressure-Targeted Cardiopulmonary Resuscitation Improves 24-Hour Survival From Ventricular Fibrillation Cardiac Arrest. Crit Care Med 44(11):e1111-e1117 doi:10.1097/CCM.0000000000001859 Nosrati R, Lin S, Mohindra R, Ramadeen A, Toronov V, Dorian P (2019) Study of the Effects of Epinephrine on Cerebral Oxygenation and Metabolism During Cardiac Arrest and Resuscitation by Hyperspectral Near-Infrared Spectroscopy. Crit Care Med 47(4):e349-e357 doi:10.1097/CCM.0000000000003640 Panchal AR, Berg KM, Hirsch KG, et al. (2019) 2019 American Heart Association Focused Update on Advanced Cardiovascular Life Support: Use of Advanced Airways, Vasopressors, and Extracorporeal Cardiopulmonary Resuscitation During Cardiac Arrest: An Update to the American Heart Association Guidelines for Cardiopulmonary Resuscitation and Emergency Cardiovascular Care. Circulation 140(24):e881-e894 doi:10.1161/CIR.0000000000000732 Paradis NA, Martin GB, Rivers EP, et al. (1990) Coronary perfusion pressure and the return of spontaneous circulation in human cardiopulmonary resuscitation. JAMA 263(8):1106-13 Perkins GD, Ji C, Deakin CD, et al. (2018) A Randomized Trial of Epinephrine in Out-of-Hospital Cardiac Arrest. N Engl J Med 379(8):711-721 doi:10.1056/NEJMoa1806842 Prengel AW, Lindner KH, Keller A (1996) Cerebral oxygenation during cardiopulmonary resuscitation with epinephrine and vasopressin in pigs. Stroke 27(7):1241-8 doi:10.1161/01.str.27.7.1241 Ristagno G, Sun S, Tang W, Castillo C, Weil MH (2007) Effects of epinephrine and vasopressin on cerebral microcirculatory flows during and after cardiopulmonary resuscitation. Crit Care Med 35(9):2145-9 doi:10.1097/01.ccm.0000280427.76175.d2 Ristagno G, Tang W, Huang L, et al. (2009) Epinephrine reduces cerebral perfusion during cardiopulmonary resuscitation. Crit Care Med 37(4):1408-15 doi:10.1097/CCM.0b013e31819cedc9 Ristagno G, Tang W, Sun S, Weil MH (2008) Cerebral cortical microvascular flow during and following cardiopulmonary resuscitation after short duration of cardiac arrest. Resuscitation 77(2):229-34 doi:10.1016/j.resuscitation.2007.12.013 Sutton RM, Friess SH, Bhalala U, et al. (2013) Hemodynamic directed CPR improves short-term survival from asphyxia-associated cardiac arrest. Resuscitation 84(5):696-701 doi:10.1016/j.resuscitation.2012.10.023 Sutton RM, Friess SH, Naim MY, et al. (2014) Patient-centric blood pressure-targeted cardiopulmonary resuscitation improves survival from cardiac arrest. Am J Respir Crit Care Med 190(11):1255-62 doi:10.1164/rccm.201407-13430C Thorup L, Koch KU, Upton RN, Ostergaard L, Rasmussen M (2019) Effects of Vasopressors on Cerebral Circulation and Oxygenation: A Narrative Review of Pharmacodynamics in Health and Traumatic Brain Injury. J Neurosurg Anesthesiol 
doi:10.1097/ANA.0000000000000596 Voelckel WG, Lurie KG, McKnite S, et al. (2000) Comparison of epinephrine and vasopressin in a pediatric porcine model of asphyxial cardiac arrest. Crit Care Med 28(12):3777-83 Voelckel WG, Lurie KG, McKnite S, et al. (2002) Effects of epinephrine and vasopressin in a piglet model of prolonged ventricular fibrillation and cardiopulmonary resuscitation. Crit Care Med 30(5):957-62 doi:10.1097/00003246-200205000-00001 Wenzel V, Krismer AC, Arntz HR, et al. (2004) A comparison of vasopressin and epinephrine for out-of-hospital cardiopulmonary resuscitation. N Engl J Med 350(2):105-13 doi:10.1056/NEJMoa025431 Wenzel V, Lindner KH, Baubin MA, Voelckel WG (2000a) Vasopressin decreases endogenous catecholamine plasma concentrations during cardiopulmonary resuscitation in pigs. Crit Care Med 28(4):1096-100 doi:10.1097/00003246-200004000-00031 Wenzel V, Lindner KH, Krismer AC, Miller EA, Voelckel WG, Lingnau W (1999) Repeated administration of vasopressin but not epinephrine maintains coronary perfusion pressure after early and late administration during prolonged cardiopulmonary resuscitation in pigs. Circulation 99(10):1379-84 doi:10.1161/01.cir.99.10.1379 Wenzel V, Lindner KH, Krismer AC, et al. (2000b) Survival with full neurologic recovery and no cerebral pathology after prolonged cardiopulmonary resuscitation with vasopressin in pigs. J Am Coll Cardiol 35(2):527-33 doi:10.1016/s0735-1097(99)00562-8 Wolfe HA, Sutton RM, Reeder RW, et al. (2019) Functional outcomes among survivors of pediatric in-hospital cardiac arrest are associated with baseline neurologic and functional status, but not with diastolic blood pressure during CPR. Resuscitation 143:57-65 doi:10.1016/j.resuscitation.2019.08.006

\section{Table}

Table 1: Comparison of the effects of the second dose of epinephrine in a.) the total cohort $(n=67), b$.) epinephrine responders $(n=47)$, and c.) epinephrine non-responders $(n=20)$.

Vasopressor effects were defined as the difference between pre-vasopressor mean and post-vasopressor maximum. Medians are reported with interquartile ranges. These differences were compared using Wilcoxon rank sum statistical tests.

$\mathrm{IQR}=$ interquartile range; $\mathrm{CoPP}=$ coronary perfusion pressure; $\mathrm{MAP}=$ mean arterial pressure; Mean $\mathrm{PA}=$ mean pulmonary artery pressure; RAP = right atrial pressure; EtCO2 = end tidal carbon dioxide; $\mathrm{CBF}=$ cerebral blood flow; $\mathrm{PbtO}_{2}=$ brain tissue oxygenation .
A. The total cohort $(n=67)$ 


\begin{tabular}{|c|c|c|c|}
\hline & Epinephrine \#2 D & Vasopressin D & $\mathbf{p}$ \\
\hline \multicolumn{4}{|c|}{ Total Cohort $(n=67)$} \\
\hline CoPP & $5.43(1.56-9.82)$ & $8.16(4.35-12.06)$ & 0.022 \\
\hline $\mathrm{CBF}$ & $0.00(-0.77-18.24)$ & $14.58(-0.05-38.12)$ & 0.005 \\
\hline MAP & $5.90(1.98-13.82)$ & $8.62(3.90-13.16)$ & 0.21 \\
\hline Mean PA & $0.04(-0.80-2.98)$ & $4.05(0.42-7.80)$ & $<0.001$ \\
\hline RAP & $0.94(0.43-1.61)$ & $0.70(0.19-1.39)$ & 0.25 \\
\hline ETCO2 & $-0.18(-2.37-2.83)$ & $-0.19(-1.5-0.98)$ & 0.74 \\
\hline \multicolumn{4}{|c|}{ Epinephrine Responders ( $n=47$ ) } \\
\hline CoPP & $6.89(2.46-10.65)$ & $8.24(4.18-13.99)$ & 0.21 \\
\hline $\mathrm{CBF}$ & $0.065(-0.51-22.67)$ & $16.03(-0.15-44.8)$ & 0.04 \\
\hline MAP & $9.00(3.32-14.62)$ & $9.02(4.40-13.99)$ & 0.60 \\
\hline Mean PA & $0.23(-0.65-2.98)$ & $4.26(0.66-8.39)$ & 0.001 \\
\hline RAP & $0.91(0.43-1.38)$ & $0.81(0.91-1.44)$ & 0.64 \\
\hline ETCO2 & $-0.02(-2.11-2.93)$ & $-0.18(-1.31-0.98)$ & 0.63 \\
\hline \multicolumn{4}{|c|}{ Epinephrine Non-responders $(n=20)$} \\
\hline CoPP & $2.76(1.28-6.58)$ & $7.64(4.86-11.02)$ & 0.22 \\
\hline $\mathrm{CBF}$ & $-0.28(-2.33-6.91)$ & $14.58(3.55-34.90)$ & 0.045 \\
\hline MAP & $2.53(0.91-7.29)$ & $6.84(3.68-10.34)$ & 0.16 \\
\hline Mean PA & $-0.13(-1.71-2.37)$ & $2.45(0.44-6.59)$ & 0.01 \\
\hline RAP & $1.19(0.49-2.05)$ & $0.70(0.22-1.29)$ & 0.23 \\
\hline ETCO2 & $-0.82(-2.86-1.29)$ & $-0.47(-1.87-0.90)$ & 0.95 \\
\hline
\end{tabular}

Figures 


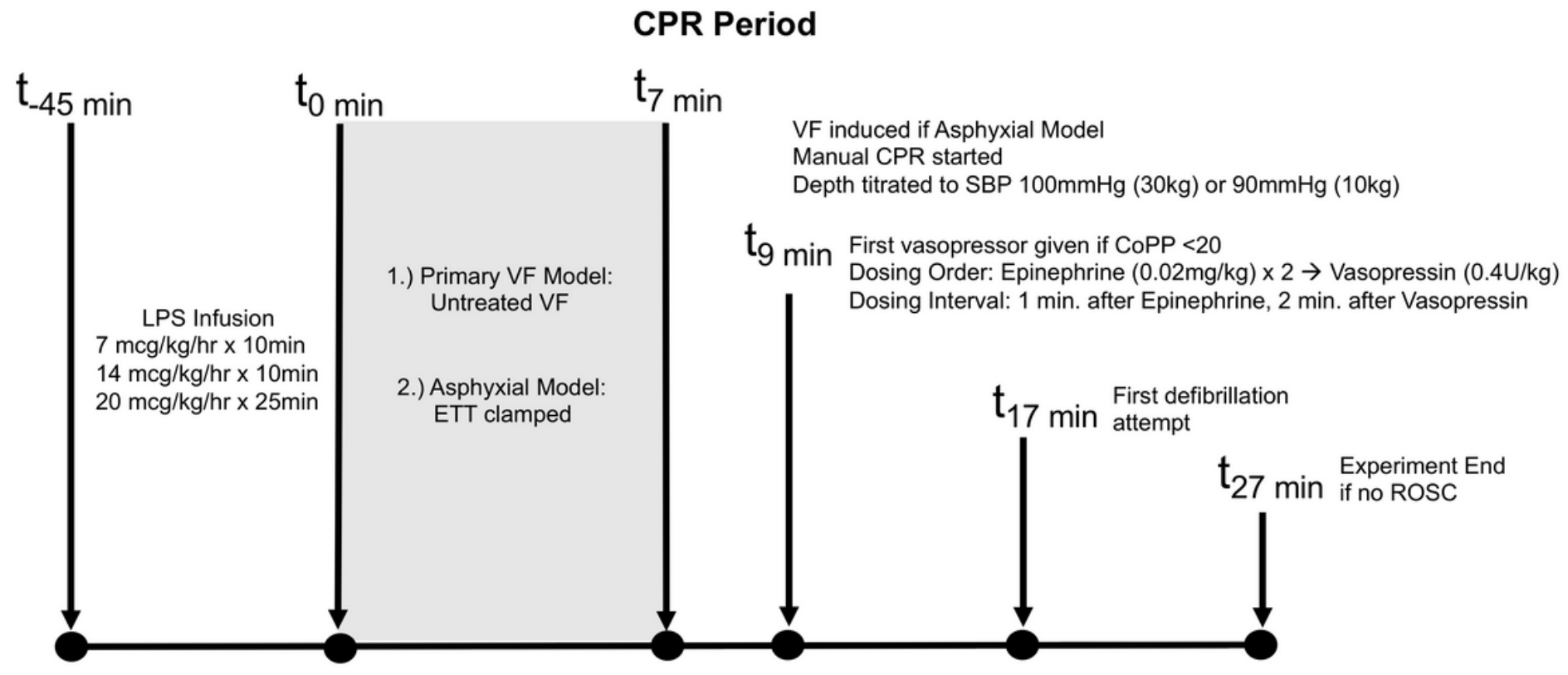

CPR (Rate $100 \mathrm{~min}^{-1}$, ventilations $6 \mathrm{~min}^{-1}$, PEEP $6 \mathrm{cmH}_{2} \mathrm{O}, 100 \% \mathrm{FiO}_{2}$ )

\section{Figure 1}

Hemodynamic-directed CPR protocol Footnote: $\mathrm{CPR}=$ cardiopulmonary resuscitation; $\mathrm{ETT}$ = endotracheal tube; $\mathrm{VF}$ = ventricular fibrillation; $\mathrm{HD}=$ hemodynamic-directed; $\mathrm{SBP}$ = systolic blood pressure; $\mathrm{CoPP}=$ coronary perfusion pressure; ROSC $=$ return of spontaneous circulation 


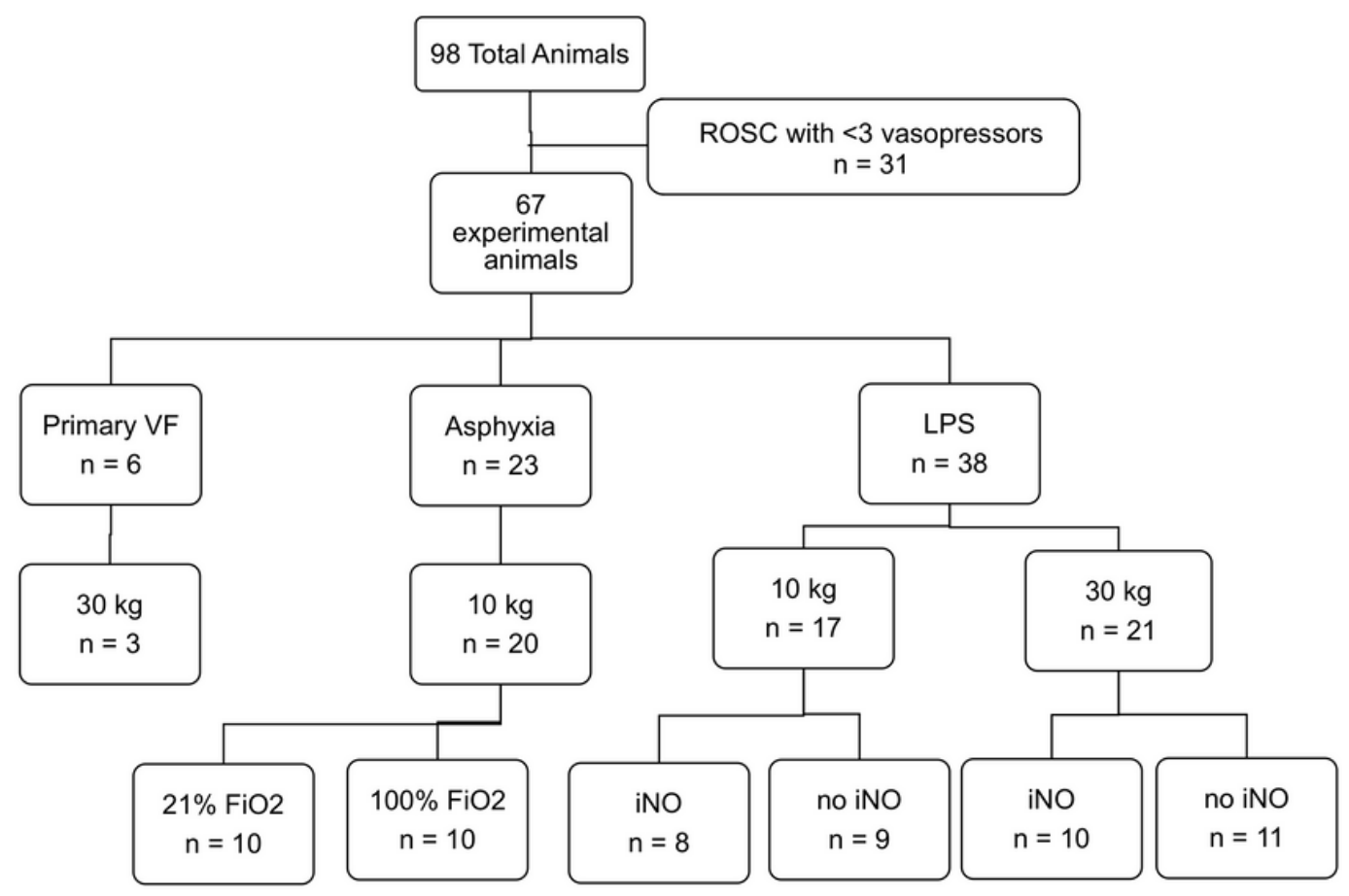

Figure 2

Study population enrollment and characteristics.

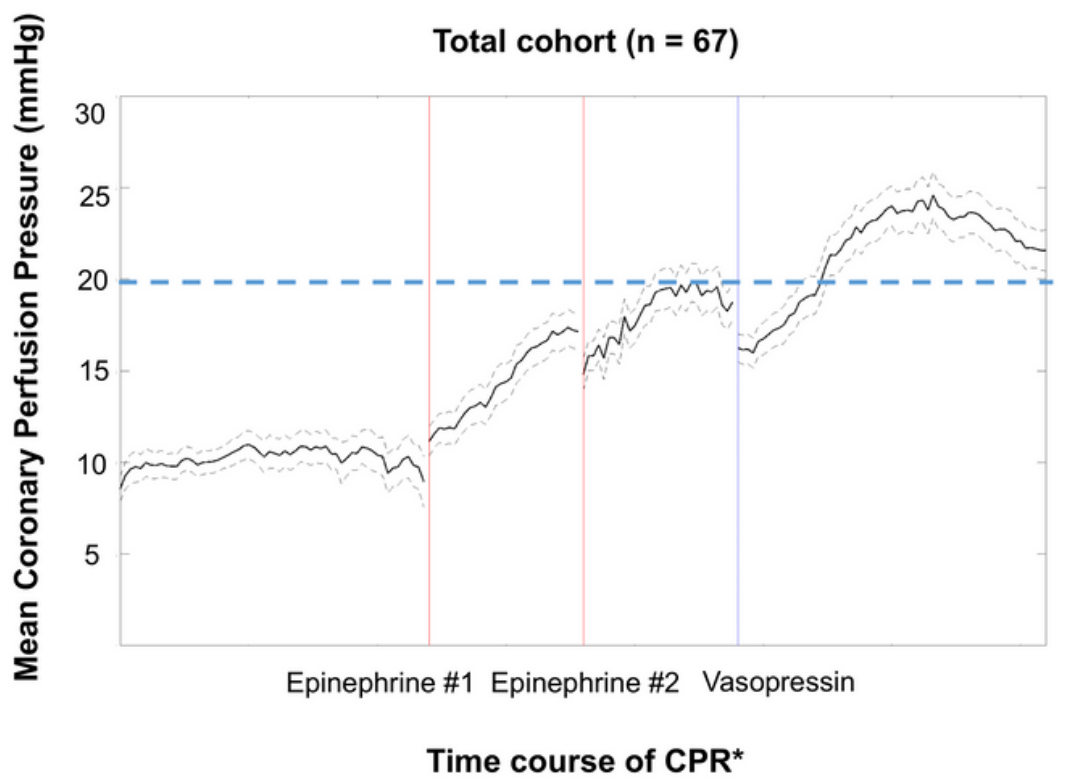




\section{Figure 3}

a: Coronary perfusion pressure during cardiac arrest in the total cohort $(n=67)$ b: Coronary perfusion pressure during cardiac arrest in a population of epinephrine non-responders $(n=20)$, defined by coronary perfusion pressure $<20 \mathrm{mmHg}$ after both doses of epinephrine. c: Coronary perfusion pressure during cardiac arrest in the population of epinephrine non-responders with subsequent response to vasopressin $(n=9)$, defined by coronary perfusion pressure $\otimes 20 \mathrm{mmHg}$ after vasopressin. ${ }^{*}$ The time course of CPR is depicted non-linearly due to the fact that vasopressors are delivered when needed as based on CoPP $<20 \mathrm{mmHg}$. The first two vertical lines represent the first and second epinephrine doses and the third vertical line represents the vasopressin dose. The two minutes before the first dose of epinephrine, then the one minute of data between vasopressors, and lastly the two minutes post-vasopressin are depicted. The dashed, horizontal line depicts the a priori CoPP goal of $20 \mathrm{mmHg}$.

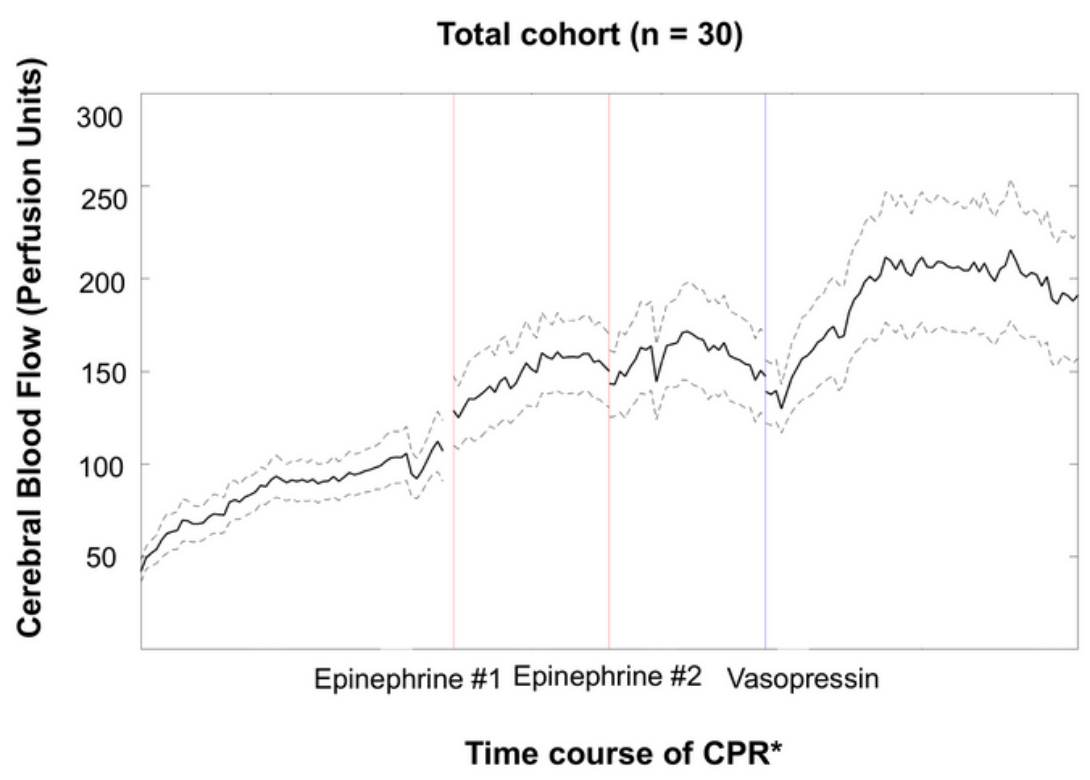

\section{Figure 4}

Cerebral blood flow during cardiac arrest in the total cohort of subjects with neuromonitoring devices in place $(n=62)$. ${ }^{*}$ The time course of CPR is depicted non-linearly due to the fact that vasopressors are delivered when needed as based on CoPP $<20 \mathrm{mmHg}$. The first two vertical lines represent the first and second epinephrine doses and the third vertical line represents the vasopressin dose. The two minutes before the first dose of epinephrine, then the one minute of data between vasopressors, and lastly the two minutes post-vasopressin are depicted.

\section{Supplementary Files}


This is a list of supplementary files associated with this preprint. Click to download.

- SupplementalTable1.docx 